\title{
Vascular occlusion to protect against intraoperative blood loss in liver surgeries: new perspectives on a traditional technique
}

\author{
Francesca Ratti ${ }^{1}$, Alessandro Nini $^{2,3,4}$, Roberto Bertini ${ }^{2,3}$, Luca Aldrighetti ${ }^{1}$ \\ ${ }^{1}$ Hepatobiliary Surgery Division, IRCCS San Raffaele Hospital, Milan, Italy; ${ }^{2}$ Department of Urology, IRCCS, San Raffaele Scientific Institute, \\ Milan, Italy; ${ }^{3}$ Division of Experimental Oncology/Unit of Urology, URI, IRCCS San Raffaele Hospital, Milan, Italy; ${ }^{4}$ Department of Urology and \\ Pediatric Urology, Universitätsklinikum des Saarlandes, Homburg, Germany \\ Correspondence to: Francesca Ratti, MD. Hepatobiliary Surgery Division, IRCCS San Raffaele Hospital, Via Olgettina 60, 20132, Milan, Italy. \\ Email: ratti.francesca@hsr.it. \\ Comment on: Shen L, Uz Z, Verheij J, et al. Interpatient heterogeneity in hepatic microvascular blood flow during vascular inflow occlusion (Pringle \\ manoeuvre). Hepatobiliary Surg Nutr 2020;9:271-83.
}

Submitted Mar 25, 2021. Accepted for publication Apr 15, 2021.

doi: 10.21037/hbsn-21-128

View this article at: http://dx.doi.org/10.21037/hbsn-21-128

The correlation between decreased blood loss and improved clinical and oncological outcomes has been described in liver surgery: considering that perioperative blood transfusions are associated with a higher rate of recurrence and lower survival after resection of colorectal liver metastases and hepatocellular carcinoma, any effort to address the task of bleeding control and reduce the need for blood transfusions is of outstanding importance (1). Blood loss and the need for transfusion during liver surgery can be decreased by several surgical adjustments or techniques, such as low central venous pressure, new haemostatic devices for parenchymal transection and control of vascular inflow and outflow (2). An effective method to control intraoperative bleeding is portal triad clamping, also known as Pringle maneuver, which can be easily performed both in open and mini-invasive hepatectomy.

Shen et al. (3) reported a heterogeneity in the effect caused by vascular occlusion by Pringle maneuver on hepatic microcirculation. In a series of 15 major hepatic resections they investigated functional capillarity density (FCD), microvascular flow index (MFI) and sinusoidal diameter (SinD) through a handleheld vital microscope, identifying two distinct populations of patients: full responders, who recorded a complete interruption of the circulation in the microvascular bed upon vascular occlusion, and partial responders, with partial persistence of the microcirculation despite clamping. In spite of this different effect of clamping, no significant differences were found in terms of blood loss between the two groups, witnessing how the Pringle maneuver is effective in protecting against bleeding even in the presence of an incomplete effect on the microcirculation. Regarding the causes of this finding, the authors trace it back to two possible explanations: the presence of a backflow from the hepatic veins and the presence of porto-systemic and arterial collateral circulation that support vascularization.

Since the preload was not measured in the study by Shen et al. (3) it is possible that the different response on microcirculation depends, at least partially, on inter-patient differences in terms of intraoperative volemic status. In fact, many reports have documented how the reduction in preload allows for a significant reduction in blood losses from the hepatic veins, thus emphasizing the importance of a proper anaesthesiological management for hemodynamic optimization in liver resections. Historically, preload monitoring has always been conducted by measuring central venous pressure (CVP), through the central venous catheter. With the large-scale introduction of the minimally invasive approach, while the need to maintain a state of hypovolemia remained unchanged-thus controlling bleeding from the hepatic side-the reliability of CVP measurement was affected by the positions of the patient and the effect of pneumoperitoneum typical of laparoscopy. Therefore, recently, non-invasive monitoring techniques have been described in hepatic surgery based on the calculation of hemodynamic parameters such as stroke volume and 
stroke volume variation, cardiac output, cardiac index and oxygen delivery. The availability of these parameters allows a very precise and accurate intraoperative management, effectively controlling the preload and therefore the hepatic bleeding (4). On the other hand, it reduces the risk of tissue hypoperfusion linked to significant hypovolemia and to the use of the Pringle maneuver and the consequent adverse effects respectively on renal function and on alterations of postoperative liver blood tests. It would therefore be interesting to validate the analysis described by Shen et al. (3) associating the microscopic study of the microcirculation with the hemodynamic evaluation: it is indeed possible that the heterogeneity of response to Pringle maneuver is linked to a different hemodynamic state of the patient (altering the tolerance to vascular occlusion), rather than to presence of collateral circulation.

Another interesting setting to study the effect of the Pringle maneuver is minimally invasive techniques: in this scenario, the biological surgical damage seems to be mitigated by the favorable biological context (5), but paradoxically the ischemia-reperfusion damage caused by Pringle's maneuver may add up to the effect of pneumoperitoneum pressure. Indeed, the haemostatic effect of pneumoperitoneum, facilitating control of potential hepatic venous bleeding, greatly contributes in obtaining a precise haemostasis and is advocated as the main determinant of blood loss reduction described in laparoscopy. The fear that these two effects may be enhanced and cause detrimental effects on the patient is the reason why vascular occlusion was initially applied with restraints in laparoscopy, to prevent a worse hepatic ischemia-reperfusion injury and consequent functional damage in cases of impaired livers. In reality, the lower biological impact of laparoscopy in terms of alteration of blood coagulation profile and lactate increase is one of the reasons for the widespread diffusion and acquirement of an established role for this technique for liver surgery, especially in "high risk" patients (6). On the other side, the effect of pneumoperitoneum may reduce the rate of non-responders to Pringle maneuver, causing a complete interruption of the microcirculation.

The concern of a potential damage especially on the fragile parenchyma (i.e., chronic liver disease and damage from chemotherapy) is the reason why control of the ischemiareperfusion damage through the application of intermittent clamping and implementing the ischemic pre-conditioning technique has traditionally been recommended. With the same rationale, the analysis of the effect of preoperative steroid administration has been extensively studied to reduce the activation of the inflammatory damage that underlies sinusoidal liver damage (7). These latest studies have often produced conflicting results and-in light of the findings of Shen et al. (3)-it is possible that this heterogeneity of results may be a consequence of a different level of "damage" which parallels the abovecited heterogeneity of effect on the microcirculation of vascular occlusion; this hypothesis needs to be validated investigating the effect of the steroid in the prevention of ischemia-reperfusion damage specifically in the population of full responders.

In conclusion, the preliminary data from Shen et al. (3) open an interesting and necessary in-depth window, aimed at investigating the effects of vascular occlusion maneuvers in some specific settings after stratification of patients according to their microcirculatory response. This would allow a further improvement in the haemodynamic management patients, a better control of the consequences of used techniques and technologies and finally an optimization of the prevention of parenchymal damage following portal clamping.

\section{Acknowledgments}

Funding: None.

\section{Footnote}

Provenance and Peer Review: This article was commissioned by the editorial office of Hepatobiliary Surgery and Nutrition. The article did not undergo external peer review.

Conflicts of Interest: All authors have completed the ICMJE uniform disclosure form (available at https://hbsn. amegroups.com/article/view/10.21037/hbsn-21-128/coif). Dr. LA serves as the unpaid editorial board member of the Hepatobiliary Surgery and Nutrition. The other authors have no conflicts of interest to declare.

Ethical Statement: The authors are accountable for all aspects of the work in ensuring that questions related to the accuracy or integrity of any part of the work are appropriately investigated and resolved.

Open Access Statement: This is an Open Access article distributed in accordance with the Creative Commons Attribution-NonCommercial-NoDerivs 4.0 International License (CC BY-NC-ND 4.0), which permits the noncommercial replication and distribution of the article with 
the strict proviso that no changes or edits are made and the original work is properly cited (including links to both the formal publication through the relevant DOI and the license). See: https://creativecommons.org/licenses/by-nc-nd/4.0/.

\section{References}

1. Pulitanò C, Arru M, Bellio L, et al. A risk score for predicting perioperative blood transfusion in liver surgery. Br J Surg 2007;94:860-5.

2. Nini A, Muttin F, Cianflone F, et al. Perioperative and oncologic outcomes of open radical nephrectomy and inferior vena cava thrombectomy with liver mobilization and Pringle maneuver for Mayo III level tumor thrombus: single institution experience. Minerva Urol Nefrol 2020. doi: 10.23736/S0393-2249.20.03844-8. [Epub ahead of print].

3. Shen L, Uz Z, Verheij J, et al. Interpatient heterogeneity in hepatic microvascular blood flow during vascular inflow occlusion (Pringle manoeuvre). Hepatobiliary Surg Nutr
2020;9:271-83.

4. Ratti F, Cipriani F, Reineke R, et al. Intraoperative monitoring of stroke volume variation versus central venous pressure in laparoscopic liver surgery: a randomized prospective comparative trial. HPB (Oxford)

2016;18:136-44.

5. Ratti F, Fiorentini G, Cipriani F, et al. Laparoscopic vs Open Surgery for Colorectal Liver Metastases. JAMA Surg 2018;153:1028-35.

6. Ratti F, Cipriani F, Reineke R, et al. The clinical and biological impacts of the implementation of fast-track perioperative programs in complex liver resections: A propensity score-based analysis between the open and laparoscopic approaches. Surgery 2018;164:395-403.

7. Pulitanò C, Aldrighetti L, Arru M, et al. Prospective randomized study of the benefits of preoperative corticosteroid administration on hepatic ischemiareperfusion injury and cytokine response in patients undergoing hepatic resection. HPB (Oxford)

2007;9:183-9.

Cite this article as: Ratti F, Nini A, Bertini R, Aldrighetti L. Vascular occlusion to protect against intraoperative blood loss in liver surgeries: new perspectives on a traditional technique. HepatoBiliary Surg Nutr 2021;10(4):567-569. doi: 10.21037/ hbsn-21-128 